\title{
ERRATUM
}

\section{Erratum for Alfano et al., Episodic Diversifying Selection Shaped the Genomes of Gibbon Ape Leukemia Virus and Related Gammaretroviruses}

\author{
Niccolò Alfano, ${ }^{a}$ Sergios-Orestis Kolokotronis,, ${ }^{\text {b,c }}$ Kyriakos Tsangaras, ${ }^{a}$ Alfred L. Roca, ${ }^{\text {d }}$ Wenqin Xu, ${ }^{\text {e Maribeth V. Eiden, }}$ \\ Alex D. Greenwood ${ }^{\text {a,f }}$ \\ Leibniz Institute for Zoo and Wildlife Research, Berlin, Germanya; Department of Biological Sciences, Fordham University, Bronx, New York, USA \\ Comparative Genomics and Division of Invertebrate Zoology, American Museum of Natural History, New York, New York, USAc; Department of Animal Sciences, \\ University of Illinois at Urbana-Champaign, Urbana, Illinois, USAd; Section on Directed Gene Transfer, Laboratory of Cellular and Molecular Regulation, National Institute of \\ Mental Health, National Institutes of Health, Bethesda, Maryland, USA ${ }^{e}$; Department of Veterinary Medicine, Freie Universität Berlin, Berlin, Germany ${ }^{f}$
}

Volume 90, no. 4, p. 1757-1772, 2016. Page 1768, Fig. 6 legend, line 1: "pol (A) and gag (B)” should read "gag (A) and pol (B).” 